\title{
Putative Clinical Implications of Unilateral Ossified Sacrospinous Ligament
}

\author{
Abu Ubaida Siddiqui ${ }^{1, \odot}$ Richa Gurudiwan² \\ ${ }^{1}$ Department of Anatomy, All India Institute of Medical Sciences, \\ Raipur, Chhattisgarh, India \\ ${ }^{2}$ Department of Anatomy, Late Shri Lakhiram Agrawal Memorial \\ Government Medical College, Raigarh, Chhattisgarh, India \\ ${ }^{3}$ Department of Cardiothoracic and Vascular Surgery, Kind Saud \\ Medical City, Riyadh, Saudi Arabia
}

| Health Allied Sci ${ }^{N U}: 2020 ; 10: 135-137$

\begin{abstract}
Address for correspondence Abu Ubaida Siddiqui, Department of Anatomy, All India Institute of Medical Sciences, Raipur 492099, Chhattisgarh, India (e-mail: anatmicro@gmail.com).
\end{abstract}

Abstract

Keywords
- sacrospinous ligament
- sacrotuberous
ligament
- compression
- ossification
- pudendal nerve

Introduction Sacrospinous ligament (SSL) is a thin structure that extends from the ischial spine to the lateral margins of sacrum and coccyx, anterior to the Sacrotuberous ligament (STL). The internal pudendal and inferior gluteal vessels, pudendal nerve, sciatic nerve, and other branches of sacral nerve plexus pass through the greater sciatic foramen (GSF) in close proximity to the ischial spine and SSL.

Objective This study aimed to report a case encountered during the routine osteology tutorial, where one of the pelvises presented with ossified SSL on the right side along with ossification of lumbosacral and the sacroiliac joints.

Case Report The SSL of the right side of the pelvis was completely ossified, extending between the ischial spine and fifth piece of sacrum. The ossification had a broadbased origin from the sacrum and tapered as in proceeded to the ischial spine. It was attached to the ischial spine with a slight enlargement. Moreover, the lumbosacral and the sacroiliac joints also appeared to be ossified.

Conclusion Ossification of the SSL can restrict the lesser sciatic foramen and the greater sciatic foramen, thus causing compression of neurovascular structures traversing these areas. This can be the causative factor in pudendal nerve/sciatic nerve entrapment and can be a cause for undiagnosed chronic perineal pain. Proper anatomical knowledge and radiologic studies can be utilized for accurate diagnosis and treatment of neurovascular compression syndromes and also during reconstructive procedures of the pelvic floor and treatment of uterovaginal prolapse.

\section{Introduction}

Sacrospinous ligament (SSL) is a thin structure which extends from the ischial spine to the lateral margins of sacrum and coccyx, anterior to the sacrotuberous ligament (STL), with which it blends in part. ${ }^{1}$ The SSL along with the STL divides the sciatic notches of the ischium and ilium into the lesser sciatic foramen (LSF) and greater sciatic foramen (GSF), respectively. The internal pudendal and inferior gluteal vessels, pudendal nerve, sciatic nerve, and other branches of sacral nerve plexus pass through the GSF in close proximity to the ischial spine and SSL. ${ }^{2}$ The coccygeus muscle has the same bony attachments and runs an identical course to the SSL. ${ }^{3}$

Several muscles, such as gluteus maximus, piriformis, biceps femoris, and various ligaments, such as anterior and posterior sacroiliac, STL, and SSL surrounding the joint, influence the movement and stability of this joint. ${ }^{4}$ An ossified STL or SSL may crush or cause lesion to the pudendal nerve when passing through the interligamentous space of these ligaments resulting in pudendal nerve entrapment. ${ }^{5} \mathrm{~A}$ case of right SSL ossification causing sciatic pain was reported in 2009 . This was due to the compression of proximal sciatic nerve due to an ossified SSL. ${ }^{6}$ It is stated that the SSL is triangular in shape and inserts 


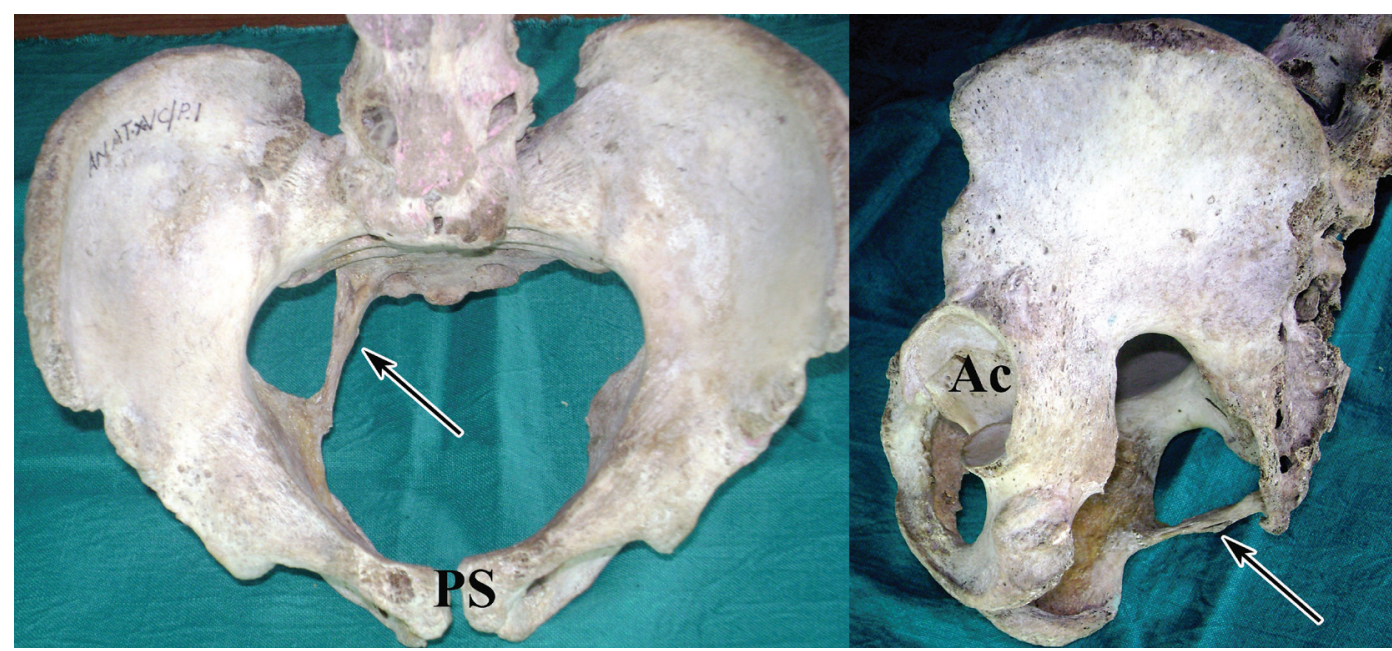

Fig. 1 Unilateral ossified sacrospinous ligament, lumbosacral joint, and the sacroiliac joints. Ac, acetabulum; PS, pubic symphysis.

on the medial edge of the sacrum, fourth and fifth vertebra, and the coccyx; runs diagonally downward, outward, and forward; and terminating at the top of the ischial spine. The SSL crosses the sciatic nerve at its distal two-thirds. ${ }^{6}$ Ossification of the STL has been reported by earlier researchers. ${ }^{7,8}$

The ossified SSL carries significance as the STL-SSL complex has a distinct function in maintaining pelvic stability and, in particular, sacroiliac joint and pubic symphysis stability. ${ }^{9}$ In an earlier report, bilateral ankylosis of sacroiliac joint with ossified sacrospinous, STL and transverse acetabular ligament was reported (Fig. 1). ${ }^{10}$ In the present case, we describe a pelvic bone with ossified unilateral SSL throughout its course.

\section{Case Report}

During the routine osteology tutorials for undergraduate medical students, one of the pelvises presented with ossified SSL. The origin, as well as gender of the bone, was unknown. The specimen was photographed and is described here. The SSL of the right side was completely ossified, extending between the ischial spine and fifth piece of sacrum. The ossification had a broad-based origin from the sacrum and tapered as in proceeded to the ischial spine. It was attached to the ischial spine with a slight enlargement. Moreover, the lumbosacral and the sacroiliac joints also appeared to be ossified.

\section{Discussion}

Ossification of the SSL can be the causative factor for restricting the anatomical areas within the LSF and GSF, thus resulting in compression of neurovascular structures traversing these areas. This can be the causative factor in pudendal nerve/sciatic nerve entrapment and can be an important cause for undiagnosed chronic perineal pain. It is stated that calcification of various soft tissues in the musculo skeletal system is a well-known phenomenon. In many cases, it may be the late sequalae of a normal damage-repair process, Calcification may affect various tissues such as synovium, muscle, and cartilage. ${ }^{11,12}$

In a study, three possible sites for pudendal nerve and vessels entrapment have been described, as it winds and passes through the fibers of SSL, between STL and SSL, and along the falciform process of STL. ${ }^{5,13}$ Such variations also possess significance in sacrospinous colpopexy, a surgical procedure that corrects vaginal vault prolapse posthysterectomy by suspending the vault to the SSL. Certain authors have advocated the placement of SSL fixation sutures in the lateral third of SSL to decrease the risk of injury to pudendal nerves. ${ }^{3}$

Moreover, it was reported in a study that in eight cases (out of 73 ), that is, $11 \%$, the inferior rectal nerve (IRN) pierces the SSL. In these cases, the IRN is prone for entrapment resulting in IRN syndrome. In this syndrome, the patient describes both perineal and perianal paresthesia which can be accompanied by anal sphincter insufficiency. ${ }^{14}$

\section{Conclusion}

The SSL remains a structure with tempting easy access. It is simply the most convenient structure for treating vaginal vault and uterine prolapse..$^{15}$ The study on ossified SSL has significance in clinical and diagnostic purposes. Proper anatomical knowhow and radiological studies can be utilized for correct diagnosis and treatment of neurovascular compression syndromes and also during the practice of reconstructive procedures of the pelvic floor and surgical treatment of uterovaginal prolapse.

\section{Conflict of Interest}

None declared. 


\section{References}

1 Standring S, Gray's Anatomy: The Anatomical Basis of Clinical Practice. 41st ed. New York, NY: Elsevier; 2016:932-934

2 Florian-Rodriguez ME, Hare A, Chin K, Phelan JN, Ripperda CM, Corton MM. Inferior gluteal and other nerves associated with sacrospinous ligament: a cadaver study. Am J Obstet Gynecol 2016;215(5):646.e1-646.e6

3 Roshanravan SM, Wieslander CK, Schaffer JI, Corton MM. Neurovascular anatomy of the sacrospinous ligament region in female cadavers: Implications in sacrospinous ligament fixation. Am J Obstet Gynecol 2007;197(6):660.e1-660.e6

4 Cohen SP. Sacroiliac joint pain: a comprehensive review of anatomy, diagnosis, and treatment. Anesth Analg 2005;101(5):1440-1453

5 Robert R, Prat-Pradal D, Labat JJ, et al. Anatomic basis of chronic perineal pain: role of the pudendal nerve. Surg Radiol Anat 1998;20(2):93-98

6 Goddyn C, Passuti N, Leconte R, Redon H, Gouin F. Sciatic nerve compression related to ossification of the sacrospinous ligament secondary to pelvic balance abnomalities. Orthop Traumatol Surg Res 2009;95(8):645-648

7 Arora J, Mehta V, Suri RK, Rath G. Unilateral partial ossification of sacrotuberous ligament: anatomico-radiological evaluation and clinical implications. Rom J Morphol Embryol 2009;50(3):505-508

8 Uruc V, Ozden R, Kalaci A, Dogramaci Y, Basarslan SK. Unilateral complete ossification of sacrotuberous ligament: an unusual cause of pudendal nerve entrapment syndrome. Neurosurg Q 2016;26(2):185-187

9 Hammer N, Höch A, Klima S, Le Joncour JB, Rouquette C, Ramezani M. Effects of cutting the sacrospinous and sacrotuberous ligaments. Clin Anat 2019;32(2):231-237

10 Sharmila BP, Devi SK. Bilateral ankylosis of sacro-iliac joint with ossified sacrospinous, sacrotuberous and transverse acetabular ligaments. Int J Anat Var 2011;4:123-127

11 Bernasconi G, Marchetti C, Reguzzoni M, Baciliero U. Synovia hyperplasia and calcification in the human TMJ disk: a clinical, surgical, and histologic study. Oral Surg Oral Med Oral Pathol Oral Radiol Endod 1997;84(3):245-252

12 Gravanis MB, Gaffney EF. Idiopathic calcifying tenosynovitis. Histopathologic features and possible pathogenesis. Am J Surg Pathol 1983;7(4):357-361

13 Loukas M, Louis RG Jr., Hallner B, Gupta AA, White D. Anatomical and surgical considerations of the sacrotuberous ligament and its relevance in pudendal nerve entrapment syndrome. Surg Radiol Anat 2006;28(2):163-169

14 Mahakkanukrauh P, Surin P, Vaidhayakarn P. Anatomical study of the pudendal nerve adjacent to the sacrospinous ligament. Clin Anat 2005;18(3):200-205

15 Rane A, Frazer M, Jain A, Kannan K, Iyer J. The sacrospinous ligament: conveniently effective or effectively convenient? J Obstet Gynaecol 2011;31(5):366-370 